\title{
INFLUÊNCIA DA DESOCLUSÃO POSTERIOR UNILATERAL NO EQUILÍBRIO CORPORAL DE RATOS
}

\author{
INFLUENCE OF UNILATERAL POSTERIOR DISOCCLUSION ON THE BODY BALANCE OF RATS
}

\author{
Tomie Nakakuki de Campos* \\ Luciana Yoshie Fukumoto ** \\ Fernando Yoshikazu Ifuko *** \\ Emílio Satoshi Hara *** \\ Hilton Sadayuki Tiba **** \\ Matsuyoshi Mori ${ }^{* * * * * * *}$
}

\section{RESUMO}

Introdução - O objetivo deste estudo é avaliar a influência da desoclusão posterior unilateral no equilíbrio corporal de ratos. Métodos - Foram utilizados vinte e quatro ratos de laboratório (Wistar) com sessenta dias de vida extra-uterina, machos, aleatoriamente divididos em dois grupos contendo doze elementos (grupo controle e grupo experimental). Após treinamento para certificação da habilidade de caminhar sobre uma trave elevada de dois metros de comprimento, os ratos não aptos a esta atividade foram eliminados do experimento. Dessa forma, nove ratos do grupo experimental sofreram desgaste da porção coronária dos dentes posteriores $\left(1^{\circ}, 2^{\circ}\right.$ e $3^{\circ}$ molares) nos hemiarcos superior e inferior do lado esquerdo, garantindo uma desoclusão posterior unilateral dos dentes. Oito ratos do grupo controle foram mantidos com a oclusão inalterada. Os dados foram analisados estatisticamente (ANOVA - $\mathrm{p} \leq 0.05$ ) por meio de dois testes distintos (Kruskal-Wallis e Friedman). Resultados - Pelo primeiro teste estatístico, verificou-se um valor nãosignificante de $\mathrm{p}=0,4152$ e, pelo segundo teste, para o grupo estudo encontrou-se $\mathrm{p}=0,1540$ e, para o grupo controle, $\mathrm{p}=0,1846$, ambos não-significantes. Conclusão - Conclui-se que a desoclusão unilateral de dentes posteriores não provoca alteraçóes significativas no equilíbrio corporal de ratos.

DESCRITORES: Oclusão dentária - Mastigação - Mobilidade dos dentes.

\section{ABSTRACT}

Introduction - The aim of this study is to evaluate the influence of unilateral posterior discclusion on the body balance of rats, during an observation period of six months. Methods: In this study, were used twenty four laboratory male rats (Wistar), with 60 days of post natal development, randomly separated into two groups (control and study group), with twelve rats in each group. At first, all rats underwent to a training period to certify the ability to walk over an elevated bar of $2 \mathrm{~m}$ length. Those inapt rats were eliminated from the study (four rats of control group and three rats of study group). Nine rats from experimental group had their coronary part of the posterior teeth worn out $\left(1^{\text {st }}, 2^{\text {nd }}\right.$ and $3^{\text {rd }}$ molars), of both maxillary and mandibular teeth, on the left side only, in order to obtain a unilateral posterior disocclusion. The control group (eight rats) did not undergo to any modification in their occlusion. Results: The results were statistically analyzed (ANOVA $\mathrm{p} \leq 0.05$ ) using two different tests (Kruskall-Wallis and Friedman). According to the first test, it was observed a non-significant value $(\mathrm{p}=0,4152)$, and the second test presented a non-significant correlation as well; for the experimental group it was observed $\mathrm{p}=0,1540$ and for the control group $\mathrm{p}=0,1846$. Conclusion: It was possible to conclude that unilateral disocclusion, induced by unilateral wear of posterior teeth, do not provoke significant modifications on the body balance of rats.

DESCRIPTORS: Dental occlusion - Mastication - Tooth mobility.

* Professora Associada do Departamento de Prótese da Faculdade de Odontologia da Universidade de Sáo Paulo (FOUSP)

** Aluna de Graduação da Faculdade de Odontologia da Universidade de São Paulo (FOUSP). Bolsista de Iniciação Cientifica da Fundação para o desenvolvimento Cientifico e tecnológico da Odontologia (FUNDECTO)

*** C.D, participante do Programa de Atualização, modalidade Investigaçăo do Departamento de Prótese da Faculdade de Odontologia da Universidade de Sáo Paulo (FOUSP)

***** Mestre e participante do Programa de Atualização, modalidade Didático do Departamento de Prótese da Faculdade de Odontologia da Universidade de Sáo Paulo (FOUSP)

****** Professor Doutor do Departamento de Prótese da Faculdade de Odontologia da Universidade de São Paulo (FOUSP) 
Campos TN, Fukumoto LY, Ifuko FY, Hara ES, Tiba HS, Mori M. Influência da desoclusão posterior unilateral no equilíbrio corporal de ratos. Revista de Odontologia da Universidade Cidade de São Paulo 2008 jan-abr; 20(1):30-6

\section{INTRODUÇÃO}

O equilíbrio corporal é definido como o estado em que todas as forças atuantes sobre o corpo estão equilibradas, tanto em posição estática, quanto dinâmica. Essa orientação espacial é mantida pela integração funcional dos sistemas vestibular (auditivo), visual e proprioceptivo (somato-sensorial) sob a coordenação geral do cerebelo (Kandel et al. $\left.{ }^{9}, 2000\right)$

Bishara et al. ${ }^{1}$ (1994) e Defabianis ${ }^{5}$ (2003) sugerem que a oclusáo pode provocar assimetria facial e, concomitantemente, induzir modificaçóes nesses sistemas vestibular, visual e proprioceptivo que, por sua vez, podem alterar o senso de equilíbrio.

Poikela et al. ${ }^{16}$, 1995; Poikela et al. ${ }^{17}$, 2000) descreveram, num experimento envolvendo coelhos, que a extração unilateral de dentes posteriores, induzindo a uma mastigação unilateral, provoca alteraçóes na simetria craniofacial, durante a fase de desenvolvimento deste, determinando uma anteriorização da fossa articular da Articulação Têmporo-Mandibular (ATM).

Além dessas alterações na ATM, outras modificações significativas podem ocorrer, como uma hiperfunção dos músculos mastigatórios ipsilateralmente ao da mastigação unilateral e conseqüente hipertrofia muscular, além de espessamento do periósteo local, desvio da linha mediana e desenvolvimento anormal da maxila e da cabeça da mandíbula (Boyd et al. ${ }^{2}$, 1987; Huggare e Raustia ${ }^{6}$, 1992; Moss e Salentijn ${ }^{13}$, 1969; Planas ${ }^{15}$, 1997)

Kohno et al. ${ }^{11}$ (1988) e Kibana et al. ${ }^{10}$ (2002) afirmaram que o esternocleidomastóideo, um dos principais músculos responsáveis pela postura da cabeça, está em sincronia com os músculos da mastigação. E comentaram que uma hiperfunção dos músculos mastigatórios, se mantida por longo período de tempo, poderia ocasionar também uma hipertrofia dos músculos posturais, determinando, dessa forma, uma inclinação da cabeça para o mesmo lado.
Apesar desses estudos terem analisado os efeitos da mastigação unilateral no sistema estomatognático, nenhum demonstrou alteraçóes no equilíbrio corporal. O propósito desse estudo é verificar os efeitos da desoclusão unilateral dos dentes posteriores no equilíbrio corporal de ratos, durante um intervalo de tempo correspondente a seis meses.

\section{MÉTODOS}

O Protocolo para uso de animais em experimentação $\left(n^{\circ} 189 / 02\right)$ da presente pesquisa está de acordo com os Princípios Éticos na experimentação animal, adotado pelo Colégio Brasileiro de Experimentação Animal (COBEA) e foi aprovado pela Comissão de Ética em Experimentaçấo Animal (CEEA) do Instituto de Ciências Biomédicas da Universidade de São Paulo.

Utilizaram-se, inicialmente, vinte e quatro ratos machos de laboratório (Wistar), com 60 dias de vida extrauterina, obtidos do Biotério da Faculdade de Medicina Veterinária e Zootecnia da USP. Os ratos foram divididos aleatoriamente em dois grupos (controle e experimental) de doze elementos. Para melhor acomodação, cada grupo foi dividido em duas gaiolas, contendo seis ratos. Os animais foram identificados por marcas nas caudas, feitas com caneta hidrofóbica (marca Pilot $\left.{ }^{\circledR}\right)$, renovadas semanalmente.

Em seguida, os ratos foram submetidos a um treinamento e condicionamento para se certificar a habilidade de caminhar sobre uma trave elevada (Figura 1), para posterior avaliação do equilíbrio corporal. $\mathrm{O}$ experimento foi conduzido nas dependências do Biotério do Departamento de Cirurgia, Prótese e Traumatologia da Faculdade de Odontologia da USP.

1- Treinamento e condicionamento sobre a trave elevada

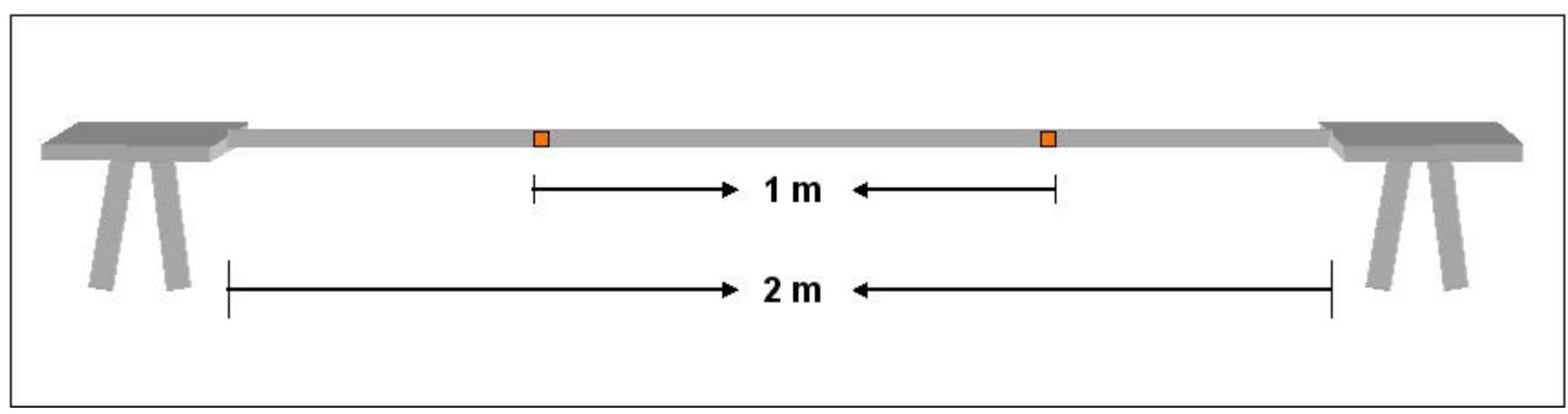

Figura 1 - Desenho esquemático da trava elevada 
Adotou-se a metodologia empregada por Rodrigues Alves $^{18}$ (2003). Primeiramente, todos os ratos foram treinados a caminhar sobre uma trave elevada, durante oito dias seguidos. O equipamento era composto de uma trave de madeira ( $18 \mathrm{~mm}$ de largura e espessura, por $2 \mathrm{~m}$ de comprimento), com uma plataforma de $10 \mathrm{~cm} \times 10 \mathrm{~cm}$, em cada uma das extremidades, apoiadas em suportes com $20 \mathrm{~cm}$ de altura. Na lateral da trave foram feitas duas marcas verticais, indicando o percurso de $1 \mathrm{~m}$, situado na porção central da trave, onde o equilíbrio corporal foi avaliado (Figura 1).

$\mathrm{O}$ treinamento consistiu, inicialmente, em colocar o rato sobre a trave elevada, assim como um reforço alimentar (leite condensado) sobre as plataformas, para atraí-lo. No primeiro dia colocou-se o rato sobre a plataforma para que se acostumasse com o ambiente e com o reforço alimentar. Nos dias subseqüentes, foi colocado cada vez mais distante da plataforma, até que apresentasse condiçóes de atravessar a trave integralmente, atingindo a plataforma oposta. Ele deveria retornar à plataforma inicial, recebendo reforço alimentar no final de cada travessia. Isso foi realizado individualmente, em sessóes de 5 minutos para cada rato.

Encerrou-se o treinamento quando o rato estava apto a caminhar sobre a trave quatro vezes, isto é, ir e vir duas vezes. Os ratos que não conseguiram caminhar sobre a trave, após o período de treinamento, foram desqualificados para a pesquisa. Contudo, foram mantidos na gaiola para manter o mesmo número de ratos em cada gaiola até a finalização do experimento.

\section{2- Desgaste dos elementos dentais posteriores}

Posteriormente ao treinamento, nove ratos do grupo experimental foram submetidos ao desgaste da porçáo coronária dos dentes posteriores (primeiro, segundo e terceiro molares) nas hemiarcadas maxilar e mandibular do lado esquerdo, garantindo uma desoclusão posterior unilateral dos dentes. Oito ratos do grupo-controle foram mantidos com a dentição inalterada.

Para o desgaste coronário, primeiramente, os ratos foram submetidos à anestesia geral por injeção intramuscular de cloridrato de quetamina de concentraçáo $10 \mathrm{mg} / \mathrm{ml}(0,01 \mathrm{ml} / 100 \mathrm{~g})$ e de cloridrato de xilazina de concentração $20 \mathrm{mg} / \mathrm{ml}(0,01 \mathrm{ml} / 100 \mathrm{~g})$.

Para o desgaste da coroa foi empregado um microscópio cirúrgico (marca D.F®. Vasconcelos, São Paulo, SP) com aumento de 16 vezes. A manobra cirúrgica de des- gaste, propriamente dita, foi realizada utilizando-se uma caneta de baixa rotaçáo e uma peça de mão, à qual foi acoplada uma broca diamantada de número 1 de peça de mão.

Após o desgaste, realizou-se o ataque ácido da superfície dentária exposta com ácido fosfórico (marca Alpha Acid ${ }^{\circledR}$, DFL, São Paulo, SP) a 37\% por 20 segundos, sucedido por lavagem da superfície dental exposta por 15 segundos, e, finalmente, aplicação de uma camada de selante resinoso fluido fotopolimerizável (marca Alpha Bond ${ }^{\circledR}$, DFL, São Paulo. SP).

Os ratos foram, então, mantidos dentro da gaiola, em temperatura ambiente, até a perda do efeito anestésico e sedativo das drogas e receberam, como alimentação, ração comercial granulada (marca Purina $\left.{ }^{\circledR}\right)$ e água "ad libitum".

Posteriormente, permaneceram em uma sala com temperatura condicionada em $20^{\circ} \mathrm{C}\left( \pm 2^{\circ} \mathrm{C}\right)$, durante todo o período da experimentação.

\section{3- Pesagem dos animais}

Os ratos foram pesados semanalmente na balança (marca Filizola ${ }^{\circledR}$, São Paulo, SP), antes do percurso na trave elevada. As pesagens foram realizadas antes do desgaste das superfícies oclusais dos dentes posteriores e após o desgaste, ao longo dos 6 meses de experimentação.

\section{4- Avaliação do equilíbrio}

$\mathrm{O}$ teste de equilíbrio foi realizado atribuindo-se escores a cada passo dado, com o membro pélvico do rato voltado para o observador, durante a caminhada do animal na porção central (um metro) da trave, entre as

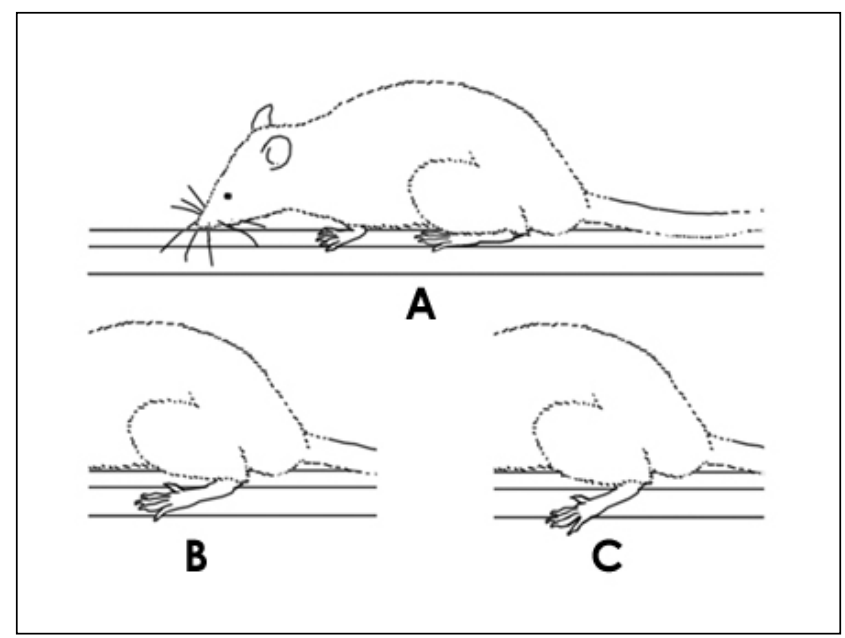

Figura 2-A) escore 0; B) escore 1; C) escore 2 
Campos TN, Fukumoto LY, Ifuko FY, Hara ES, Tiba HS, Mori M. Influência da desoclusão posterior unilateral no equilíbrio corporal de ratos. Revista de Odontologia da Universidade Cidade de São Paulo 2008 jan-abr; 20(1):30-6

marcaçóes verticais (Figura 1). Os escores utilizados foram os de Rodrigues Alves ${ }^{18}(2003)$ e estáo ilustrados na Figura 2:

$\boldsymbol{O}$ (zero) - normal (quando o membro posterior estiver completamente localizado sobre a parte superior da trave);

1 (um) - quando parte do membro posterior escorregar lateralmente à trave, sem ultrapassar o limite inferior lateral da trave;

2 (dois) - quando parte do membro posterior ultrapassar o limite inferior lateral da trave.

Os testes de equilíbrio foram realizados antes do desgaste da parte coronária dos elementos dentários e após 3 e 6 meses.

\section{RESULTADOS}

As tabelas 1 e 2 mostram os escores de cada rato, assim como a mediana dos grupos controle e experimental, durante o período de seis meses do experimento:

As medianas dos escores obtidos durante o período de análise podem ser melhor observados no Gráfico 1 .

Para a análise estatística, considerou-se como nível de significância $\alpha<0,05$ e $\beta<0,2$. Utilizou-se ANOVA (Análise de Variância) por meio de dois testes distintos para certificação de eventuais variâncias. Primeiramente, pelo Teste de Kruskal-Wallis verificou-se um valor não significante de $p=0.4152$. E pelo Teste de Friedman, encontrou-se dentro do grupo de estudo, um valor de
Tabela 1 - Grupo Experimental: escores individuais e medianas, antes do desgaste dental, após 3 e 6 meses

\begin{tabular}{cccc}
\hline \hline & Inicio & 3 meses & 6 meses \\
\hline Rato 1 & 9 & 22 & 20 \\
Rato 2 & 14 & 9 & 5 \\
Rato 3 & 20 & 1 & 9 \\
Rato 4 & 8 & 3 & 5 \\
Rato 5 & 21 & 15 & 5 \\
Rato 6 & 12 & 7 & 4 \\
Rato 7 & 4 & 8 & 12 \\
Rato 8 & 6 & 4 & 5 \\
Rato 9 & 7 & 2 & 5 \\
Medianas & 9 & 7 & 5 \\
\hline \hline
\end{tabular}

Tabela 2 - Grupo-Controle: escores individuais e medianas, antes do desgaste dental, após 3 e 6 meses

\begin{tabular}{cccc}
\hline \hline & Inicio & 3 meses & 6 meses \\
\hline Rato 1 & 26 & 22 & 13 \\
Rato 2 & 5 & 4 & 4 \\
Rato 3 & 7 & 9 & 11 \\
Rato 4 & 12 & 5 & 10 \\
Rato 5 & 10 & 9 & 7 \\
Rato 6 & 17 & 7 & 8 \\
Rato 7 & 26 & 21 & 14 \\
Rato 8 & 5 & 5 & 3 \\
Medianas & 11 & 8 & 9 \\
\hline \hline
\end{tabular}

Gráfico 1 -

Medianas dos escores dos grupos controle e de estudo, em função da duração do experimento

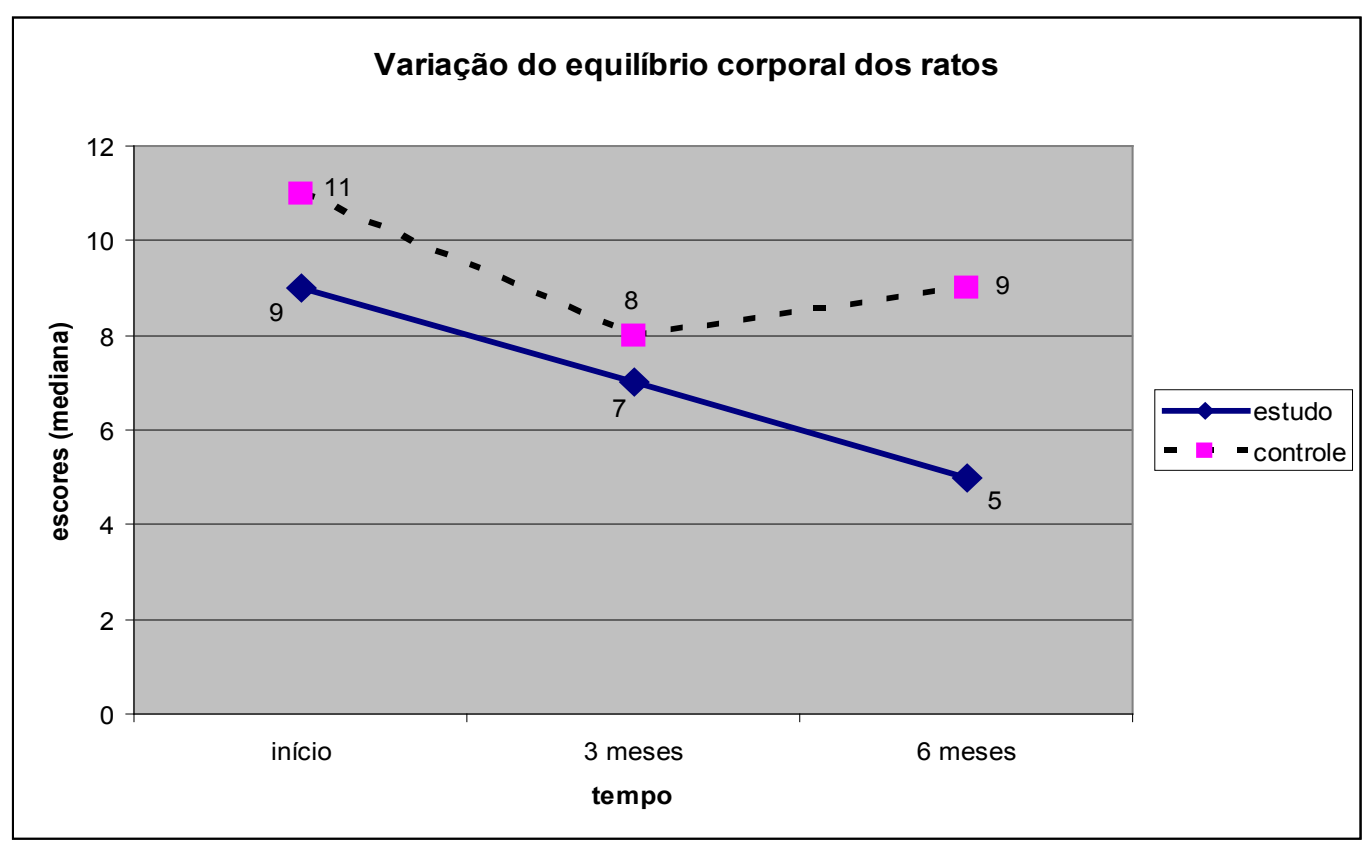




\section{Gráfico 2 -}

Médias dos pesos dos ratos dos grupos-controle e de estudo, ao longo dos 6 meses de experimentação

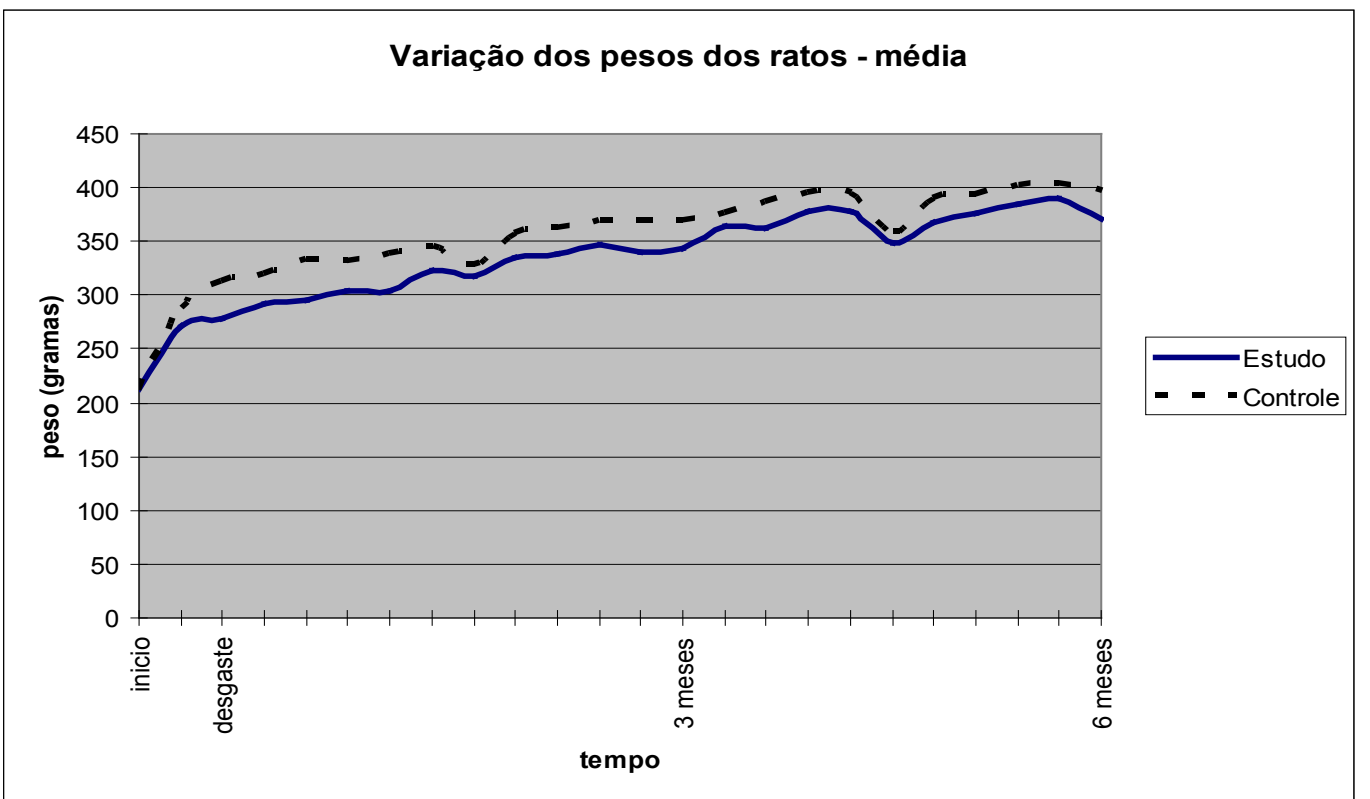

$p=0.1540$, e dentro do grupo-controle, obteve-se o valor de $p=0.1846$; ambos não significantes.

O Gráfico 2 mostra a evolução dos pesos dos ratos ao longo dos seis meses de experimentação, desde o peso inicial, sem o desgaste dos dentes, em seguida com o desgaste, com pesagens realizadas semanalmente.

\section{DISCUSSÃO}

O equilíbrio corporal é definido como o estado em que todas as forças agindo sobre o corpo estão equilibradas, devido à integração funcional dos sistemas: vestibular (auditivo), visual e proprioceptivo (somato-sensorial), sob a coordenaçáo do cerebelo (Kandel et al. ${ }^{9}, 2000$ ).

Questiona-se se fatores que possam alterar os sentidos da audição, visão e propriocepção, tais como alteraçôes morfológicas da anatomia craniofacial, poderiam causar também alteraçóes no senso de equilíbrio.

A mastigação unilateral é relatada na literatura como um fator de alteração da morfologia craniocervical, assim como um fator predisponente das Disfunções Temporomandibulares (DTMs) (Miyake et al. ${ }^{12}$, 2004), uma vez que há uma sobrecarga e concomitante ação de microtraumas na região posterior da ATM contralateral ao lado de trabalho (Huang et al. ${ }^{7}, 2002$; Huang et al. ${ }^{8}, 2003$; Naeije e Hofman ${ }^{14}$, 2003). Além disso, há uma hiperatividade muscular ipsilateral dos músculos da mastigação e também dos músculos posturais, que estáo sincronizados.

Há um interesse crescente no relacionamento da oclusão com a postura corporal, uma vez que a maioria dos pacientes com DTM relata também dores nas regióes de pescoço e tronco. A fim de estabelecer uma relação entre as condições do sistema estomatognático e a postura corporal, Yoshino et al. ${ }^{21}$ (2003) avaliaram alteraçóes na posição da cabeça durante a oclusão dos dentes, em função das perdas de apoio oclusal posterior uni e bilaterais. Pelos resultados obtidos, os autores concluíram que a perda de apoio oclusal unilateral poderia causar a desarmonia dos músculos do pescoço e, portanto, afetar a postura corporal. D'Attilio et al. ${ }^{3}$ (2005) delinearam uma pesquisa para constatar a possibilidade de induzir experimentalmente alteraçóes no alinhamento da coluna vertebral, em ratos, como conseqüência de alteração na oclusão dental. Os resultados mostraram que todos os ratos do grupo experimental desenvolveram curvas escorióticas e nenhuma alteração naqueles do grupo-controle. Os autores concluíram que o alinhamento da coluna vertebral parece influenciado pela oclusão dental.

Essas funçóes irregulares poderiam criar, a longo prazo, uma série de sinais e sintomas de DTM, inclusive alteraçôes na assimetria facial e alteraçóes na morfologia óssea (Boyd et al. ${ }^{16}, 1987$; Dawson ${ }^{4}$, 1993; Huggare e Raustia ${ }^{6}$, 1992; Moss e Salentijn ${ }^{13}$, 1969; Planas ${ }^{15}$, 1997).

As alteraçóes na ATM, principalmente as que induzem à disfunção da ATM, são relacionadas com alteraçóes no sistema auditivo, como "sensação de ouvido tapado" ou "zumbido no ouvido" (Sessle et al. $\left.{ }^{19}, 1995\right)$. 
Campos TN, Fukumoto LY, Ifuko FY, Hara ES, Tiba HS, Mori M. Influência da desoclusão posterior unilateral no equilíbrio corporal de ratos. Revista de Odontologia da Universidade Cidade de São Paulo 2008 jan-abr; 20(1):30-6

Além disso, Sharifi Milani et al..$^{20}$ (1998) afirmaram que uma alteração na oclusão também pode provocar alteraçóes no sistema visual do individuo. Entretanto, apesar desses estudos mostrarem certas alteraçóes anatômicas e alteraçôes nos sistemas responsáveis pelo equilíbrio corporal, pelos resultados apresentados na presente pesquisa, a hipótese nula não pode ser descartada, isto é, não se observou nenhuma relação significativa entre a desoclusão posterior unilateral e o equilíbrio corporal dos ratos.

Todavia, isso não rejeita a hipótese de ocorrência em seres humanos, uma vez que os sistemas de equilíbrio e de locomoção dos humanos são diferentes dos apresentados pelos animais quadrúpedes. Porém, neste trabalho, selecionou-se o rato, por ser o animal mais utilizado em experimentos, citados na literatura mundial, para avaliação da postura e equilíbrio corporal, podendo ser considerado como padrão para tais tipos de experimento.

Outro fator relevante, relacionado à anatomo-fisiologia da mastigação dos ratos, é que eles utilizam os dentes incisivos por mais tempo durante a mastigação e, quando o alimento está praticamente triturado, os molares finalizam a mastigação. Talvez seja necessária também a extração do incisivo central ipsilateral, para caracterizar de forma mais adequada a mastigação unilateral. Contudo, neste experimento, não se planejou a extração dos dentes, posteriores ou anteriores, para que o trauma do procedimento cirúrgico não interferisse nos resultados, direta ou indiretamente. Optou-se pelo desgaste coronário, que resultou na desoclusão e ausência de apoio dental posterior.

Os resultados da pesagem dos animais, em semanas alternadas, durante o período do experimento, demonstraram que os animais alimentaram-se da mesma forma que os do grupo-controle. Pelo Gráfico 2, pode-se observar que, em geral, os pesos dos ratos sofreram leves alterações no decorrer do tempo, porém, apresentando sempre resultantes crescentes, eliminando-se qualquer hipótese de má alimentação ou subnutrição dos ratos experimentais.

Para que se possa afirmar categoricamente a influência ou não da mastigação unilateral no equilíbrio corpo- ral, futuros estudos serão necessários em humanos, por meio de pesquisas clinicamente controladas, ou em animais, que apresentem maior similaridade. Nesta questão, ressalte-se que a utilização de símios em pesquisa é complicada, desde a obtenção dos animais, a aprovação pelo comitê de ética, até a necessidade de biotério e técnicos especializados na manutenção desses animais. Além disso, o período de desenvolvimento das estruturas anátomo-funcionais dos macacos é superior aos dos ratos, o que demandaria anos de pesquisa.

$\mathrm{O}$ fato do presente estudo não ter confirmado a hipótese da influência da desoclusão posterior unilateral no equilíbrio corporal em ratos, possibilitou uma análise crítica da metodologia, apontando possíveis fatores, que não são considerados nos artigos nacionais e internacionais, que também utilizam ratos. Acreditamos que a principal contribuição desta pesquisa não está na conclusão, mas sim na discussão, que gerou importantes questionamentos, para o aprimoramento desta linha de pesquisa, principalmente, quando se deseja transportar os resultados obtidos em animais para os humanos.

\section{CONCLUSÃO}

Com base na metodologia empregada, concluiu-se que a desoclusão unilateral de dentes posteriores de ratos não provoca alterações significativas no equilíbrio corporal.

\section{AGRADECIMENTOS}

À Fundação para o Desenvolvimento Tecnológico e Científico da Odontologia (FUNDECTO) pela concessão da Bolsa de Iniciação Científica.

À Profa titular Helenice de Souza Spinosa, do Departamento de Patologia da Faculdade de Medicina Veterinária e Zootecnia da USP.

Ao Prof. titular Jarbas Arruda Bauer, do Departamento de Histologia e Embriologia do Instituto de Ciências Biomédicas da USP.

Ao Prof. Associado João Gualberto de Cerqueira Luz, responsável pelo Biotério do Departamento de Cirurgia, Prótese e Traumatologia Maxilofaciais da Faculdade de Odontologia da USP. 


\section{REFERÊNCIAS}

1. Bishara SE, Burkey PS, Kharouf JG. Dental and facial asymmetries: a review. Angle Orthod 1994;64(2):8998.

2. Boyd CH, Slagle WF, Boyd CM, Bryant RW, Wiygul JP. The effect of head position on electromyographic evaluations of representative mandibular positioning muscle groups. Cranio 1987 Jan; 5(1): 50-4.

3. D'Attilio M, Filippi MR, Femminella B, Festa F, Tecco $\mathrm{S}$. The influence of an experimentally-induced malocclusion on vertebral alignment in rats: a controlled pilot study. Cranio 2005 April; 23(2):119-29.

4. Dawson PE. Avaliação, diagnóstico e tratamento dos problemas oclusais $2^{a}$ ed. Artes Médicas; 1993.

5. Defabianis P. Biology and mechanics of facial asymmetries in children and youths. Funct Orthod 2003 Spring-Summer; 20(1-2):32-9.

6. Huggare JA, Raustia AM. Head posture and cervicovertebral and craniofacial morphology in patients with craniomandibular dysfunction. Cranio 1992 Jul; 10(3): 173-7, discussion 178-9.

7. Huang Q, Opstelten D, Samman N, Tideman H: Experimentally induced unilateral tooth loss: histochemical studies of the temporomandibular joint. $J$ Dent Res 2002 Mar; 81(3):209-13.

8. Huang Q, Opstelten D, Samman N, Tideman H: Experimentally induced unilateral tooth loss: expression of type II collagen in temporomandibular joint cartilage. J Oral Maxillofac Surg 2003 Sep; 61(9):1054-60.

9. Kandel ER, Schwartz JH, Jessel TM: Posture. In: Principles of neural science. 2000. $2^{\text {nd }}$ ed. Chapter 41, p. 816-831.

10. Kibana Y, Ishijima T, Hirai T. Occlusal support and head posture. J Oral Rehabil 2002 Jan; 29(1):58-63.

11. Kohno S, Yoshida K, Kobayashi H. Pain in the sternocleidomastoid muscle and occlusal interferences. J. Oral Rehabil. 1988 Jul; 15(4):385-92.
12. Miyake R, Ohkubo R, Takehara J, Morita M. Oral prafunctions and association with symptoms of temporomandibular disorders in Japanese university students. J Oral Rehabil 2004 Jun; 6(31):518-23.

13. Moss ML, Salentijn L. The primary role of functional matrices in facial growth. Am J Orthodont 1969 Jun; 55(6): 566-77.

14. Naeije M, Hofman N. Biomechanics of the human Temporomandibular Joint during chewing. J Dent Res 2003 Jul; 82(7):528-31.

15. Planas P. Reabilitação neuroclusal. $2^{a}$ ed. Rio de Janeiro: Medsi; 1997.

16. Poikela A, Kantomaa T, Tuominen M, Pirttiniemi P. Effect of unilateral masticatory function on craniofacial growth in the rabbit. Eur J Oral Sci 1995 Apr; 103(2 ( Pt 1)):106-11.

17. Poikela A, Pirttiniemi P, Kantomaa T.: Location of the glenoid fossa after a period of unilateral masticatory function in young rabbits. Eur J Orthod 2000 Apr; 22(2):105-12.

18. Rodrigues Alves PSB. Efeitos da moxidectina no comportamento sexual de ratos machos. [Mestrado]. São Paulo: Instituto de Psicologia da Universidade de São Paulo; 2003.

19. Sessle BJ, Bryant PS, Dionne RA. Temporomandibular Disorders and related pain conditions. Seatle, USA: IASP, 1995. v.4.

20. Sharifi Milani R, Deville de Periere D, Micallef JP. Relationship between dental occlusion and visual focusing. Cranio 1998 Apr; 16(2):109-18.

21. Yoshino G, Higashi K, Nakamura T. Changes in head position due to occlusal supporting zone loss during clenching. Cranio 2003 Apr; 21(2):89-98.

Recebido em: 22/08/06 Aceito em: 18/11/07 